\title{
AVALIAÇÃO DO USO DE EXTRATO DE TIMO (TIMULINA) EM PACIENTES COM NEOPLASIA MALIGNA SUBMETIDOS AO TRATAMENTO CIRÚRGICO
}

\author{
EVALUATION OF THE USE OF THYMUS EXTRACT (TIMULINA) IN PATIENTS WITH \\ NEOPLASIC DISEASE SUBMITTED TO THE SURGICAL TREATMENT
}

\author{
Célia Regina de Oliveira Garritano, TCBC-RJ'
}

\begin{abstract}
RESUMO: Objetivo: Avaliar a ação da timulina em pacientes com neoplasia maligna, submetidos ao tratamento, com e sem quimioterapia e radioterapia complementar (QT/RT). Método: Estudo retrospectivo realizado em 50 pacientes, analisando as variações das taxas de leucócitos, linfócitos totais e da relação de linfócitos CD4/CD8 após imunoestimulação com timulina. Resultados: No grupo submetido à QT/RT ocorreu um aumento do número de leucócitos após seis meses em 43,33\% dos casos, e em 83,33\% após 12 meses. Com relação aos linfócitos totais, após seis meses, 63,33\% apresentaram níveis maiores, e depois de 12 meses isto ocorreu em casos $90 \%$ dos casos. A relação de linfócitos CD4/CD8 mostrou um aumento em 66,66\%, e em $90 \%$ depois após 6 e 12 meses respectivamente. A análise estatística se mostrou significante com o teste de ANOVA one way. No grupo não submetido à QT/RT a elevação dos níveis de leucócitos ocorreu em 85\% dos pacientes aos seis meses e em $90 \%$ aos 12 meses. As taxas de linfócitos se elevaram em $60 \%$ dos casos em seis meses e em $85 \%$ após 12 meses. A relação CD4/CD8 se tornou maior tanto aos seis como aos 12 meses em 65\%. A análise estatística mostrou relevância com o teste " $t$ " de Student e o de ANOVA one way. Não houve necessidade de interrupção dos ciclos de QT/RT e nenhum paciente referiu intolerância à timulina. Conclusão: O uso da timulina foi capaz de restaurar a resposta imune, reduzir os danos imunossupressores e colaterais induzidos pela terapia antineoplásica e não apresentou efeitos colaterais (Rev. Col. Bras. Cir. 2007; 34(4): 225-231).
\end{abstract}

Descritores: Extratos de timo; Neoplasias; Antineoplásicos.

\section{INTRODUÇÃO}

Nos trabalhos pioneiros em Imunologia, a imunidade achava-se associada apenas com a proteção do indivíduo contra agentes infecciosos. Com o maior conhecimento da biologia molecular, celular e imunogenética, o sistema imune passou a ser relacionado também à homeostase, vigilância e tolerância imunológica.

Para que ocorra uma resposta imune satisfatória, há necessidade de mecanismos específicos que dependem de uma exposição prévia (processo infeccioso, imunização ativa ou passiva) e inespecíficos (mecanismos inatos que atuam após a exposição inicial a substâncias estranhas).

Os linfócitos $\mathrm{T}$ são os responsáveis pela resposta celular, que envolve o contato célula a célula com os diversos patógenos. Neste caso entram em ação os linfócitos "helper" (CD4), supressores (CD8), citotóxicos (CD8), células "killers" (K) e "natural killers" (NK), além de macrófagos e monócitos. Os linfócitos T originam-se na medula óssea, amadurecem no timo, e durante o seu desenvolvimento os marcadores celulares específicos de superfície são inseridos para identificar cada tipo de célula ${ }^{1}$. Todo este processo é controlado pelos hormônios tímicos e seus produtos celulares (interleucinas e interferons).
A primeira referência da importância do timo na resposta imune foi feita por White em 1949, sendo que somente na década de 60 foi isolada a fração Timosina por Trainin ${ }^{2}$.

Os extratos tímicos são compostos de três diferentes hormônios ativos isolados do timo, que são a timulina (fator tímico sérico ou FTS), a timopoietina e a timosina a-1. A timulina é um peptídeo hormonal obtido a partir de um lisado ácido do timo de vitelo, que atua nas células T, B, $N K$ modulando a proliferação e diferenciação das células progenitoras da medula óssea. A timopentina é um derivado sintético da timopoietina, que promove a diferenciação e proliferação das células $\mathrm{T}$ e a produção de interleucinas. Os melhores resultados do uso do extrato tímico são obtidos sobre a imunidade celular, já tendo sido demonstrado que ele é capaz de modular a produção, maturação e ativação dos linfócitos $\mathrm{T}$ e macrófagos, além de estimular a conversão dos timócitos imaturos em linfócitos T3 na medula óssea humana ${ }^{3}$. Nas células maduras ele aumenta o número e a função dos linfócitos T4, a resposta à concavalina $\mathrm{A}$ e à fitohemoaglutinina ${ }^{3}$, a hemopoiese, a produção de anticorpos, a reatividade aos mitógenos tipo T, a competência de linfócitos T para participar de culturas mistas, o efeito "killer" contra as células tumorais, a secreção de interleucinas pelos linfócitos $\mathrm{T}^{4-7}, \mathrm{o}$ desenvolvimento da imunidade celular ${ }^{2,6}$.

1. Professora Adjunto do Departamento de Cirurgia Geral e Especializada da Universidade do Rio de Janeiro (UNI RIO); Mestre em Cirurgia Gastroenterológica pela Universidade Federal Fluminense (UFF).

Recebido em 16/01/2007

Aceito para publicação em 20/03/2007

Conflito de interesses: nenhum

Fonte de financiamento: nenhuma

Trabalho realizado no Hospital Universitário Gaffrée e Guinle, Rio de Janeiro- RJ. 
É descrito que a resposta imune é afetada pela ingestão inadequada de alimentos e de álcool em excesso, traumas físicos e emocionais, tabagismo, uso de drogas (esteróides, narcóticos, barbitúricos, antibióticos), quimioterapia, radioterapia, cirurgia, doença crônica ${ }^{8-12}$.

O uso de imunomoduladores visa a recuperação do sistema imunológico minimizando os efeitos colaterais de diversos tratamentos, além de garantir a proteção do indivíduo contra vários agentes de diferentes origens. Inúmeras referências mostram os resultados satisfatórios dos hormônios tímicos sobre as doenças de origem viral ${ }^{7-12}$, neoplásicas ${ }^{13-19}$, imunossupressoras ${ }^{17-22,23-29}$, além da ação na resposta imunológica ${ }^{1,2,3,14,30}$. O objetivo do trabalho é avaliar se há restauração da resposta imunológica de pacientes imunossuprimidos após estimulação com a timulina.

\section{MÉTODO}

Este trabalho foi realizado no Hospital Universitário Gaffrée e Guinle, no Rio de Janeiro, em 50 pacientes portadores de neoplasia maligna, comprovada por exame histopatológico de peça cirúrgica, sendo que 30 foram submetidos á quimioterapia e/ou radioterapia complementar (QT/RT).

$\mathrm{O}$ estudo foi submetido e aprovado pela Comissão de Ética em Pesquisa de acordo com a Resolução 196/96.

A imunoestimulação foi feita utilizando a timulina fornecida pelo Laboratório Extratos Alergênicos Ltda, e registrada no Ministério da Saúde com o número 1179.0005.001-1. A timulina é um polipeptídeo dialisado de peso molecular inferior a 10.000 daltons, obtido do timo de vitelo e preparado segundo o método de Goldstein ${ }^{30,31}$. A substância foi administrada por via subcutânea, na dose de $2 \mathrm{mg} / \mathrm{ml}$, conforme o esquema:

- Linfócitos totais até $500 / \mathrm{mm}^{3}=$ de 3 em 3 dias, por três meses.
- Linfócitos totais entre 500 e $1000 / \mathrm{mm}^{3}=5$ em 5 dias, por três meses.

- Linfócitos totais acima de $1000 / \mathrm{mm}^{3}=\operatorname{de} 7$ em 7 dias, por três meses.

Todos os pacientes realizaram hemograma completo e a avaliação da relação de linfócitos CD4/CD8 antes de começar o tratamento basal, os exames foram repetidos a cada três meses, para a avaliação da dose a ser aplicada, até completar 12 meses. Foram considerados para o trabalho os resultados basal, 6 e 12 meses dos leucócitos, linfócitos totais e relação de linfócitos CD4/CD8.

Para a análise estatística nós utilizamos o software Graph Pad Instat versão 3.0, San Diego Califórnia ${ }^{\circledR}$ e, para efeito de interpretação, o limite de erro tipo I foi até $5 \%(\mathrm{p} \leq 0,05)$. Os resultados foram apresentados como média \pm desvio padrão. As variáveis foram testadas pelo método Kolmogorov and Smirnov $(K S)$, a inferência estatística foi feita pelos testes " $t$ " de Student de amostras independentes e pelo teste ANOVA one way, para as três fases do tratamento, além do pós-teste de Tukey-Kramer.

\section{RESULTADOS}

As principais características dos pacientes estão relacionadas na Tabela 1

Com relação à análise estatística, todas as amostras apresentaram uma distribuição normal pelo método de Kolmogorov e Smirnov.

Avaliando os valores basais (antes do uso da timulina) dos leucócitos dos dois grupos, com e sem QT/RT, obtivemos as taxas médias, respectivamente, de 5325,3 $( \pm 1259,78)$ e 6043,5 $( \pm 1753,35)$. A análise pelo teste " $t$ " de Student mostrou $\mathrm{p}=0,09$, não sendo, portanto, significativo.

Analisando as variações ocorridas nas taxas dos leucócitos dos pacientes submetidos à QT/RT após seis me-

Tabela 1 - Características gerais da amostra estudada.

\begin{tabular}{lc}
\hline Características & Valor extraído da amostra de 50 indivíduos \\
\hline Sexo masculino & $26 \%$ \\
Sexo feminino & $74 \%$ \\
\hline Idade & $54,06 \pm 12,93(55)$ \\
& Variação de 28 a 77 anos \\
\hline Tipo de neoplasia & \\
Melanoma & $26 \%$ \\
Mama & $28 \%$ \\
Colon & $26 \%$ \\
Estômago & $8 \%$ \\
Pâncreas & $4 \%$ \\
Outros & $6 \%$ \\
2 tumores concomitantes & $2 \%$ \\
Cirurgia & $100 \%$ \\
Com QT/RT & $60 \%$ \\
Sem QT/RT & $40 \%$ \\
\hline
\end{tabular}

Valores expressos em média \pm desvio padrão e (mediana). 
ses de uso do imunoestimulante, observamos que 13 pacientes $(43,33 \%)$ apresentaram aumento do número de células, embora a média geral tenha reduzido $0,25 \%$ em relação ao valor médio basal. Entretanto, após 12 meses de tratamento, 25 pacientes $(83,33 \%)$ apresentaram uma expressiva elevação do número leucócitos, representando um aumento de 22,39\% em comparação ao valor médio inicial. A análise estatística com o teste ANOVA one way mostrou significância destes resultados, e foram confirmados pelo pós-teste de comparações múltiplas de Tukey-Kramer.

No Grupo que não fez uso de QT/RT 17 pacientes (85\%) apresentaram elevação das taxas das células aos seis meses e $18(90 \%)$ aos 12 meses, em comparação ao valor basal. Isto representou um aumento médio dos leucócitos de 9,20\% e $20,63 \%$, respectivamente em seis e 12 meses. A análise estatística destes dados se mostrou significante após 12 meses e na comparação entre 6 e 12 meses com o teste " $t$ " de Student, mas não com o de ANOVA one way. A Tabela 2 mostra as variações e análises estatísticas dos valores de leucócitos nos grupos avaliados.

A mesma análise foi realizada com as taxas dos linfócitos totais, e assim como na amostra anterior, os valores médios basais não mostraram diferença significativa $(\mathrm{p}>0,05)$ no teste " $t$ " de Student, quando comparamos os grupos submetidos e não submetidos à QT/RT.

Nos resultados do grupo que fez QT/RT, observamos que após seis meses de uso de extrato de timo, 19 pacientes $(63,33 \%)$ apresentaram valores maiores de linfócitos totais do que os iniciais, enquanto que depois de 12 meses isto ocorreu com 27 casos (90\%). Estes dados correspondem a uma elevação dos valores médios de 6,93\% nos primeiros seis meses e de $32,41 \%$ aos 12 meses, em comparação com a taxa média basal de linfócitos. A análise estatística mostrou ser bastante significativa com o teste ANOVA one way sendo confirmada pelo pós-teste de TukeyKramer.

Ainda com relação aos linfócitos totais, analisando os dados do grupo que não foi submetido à QT/RT, observamos que a elevação dos valores das células ocorreu em 12 pacientes (60\%) depois de seis meses e em 17 (85\%) aos 12 meses de uso de timulina. Além disto, em dois casos (10\%) este aumento foi maior do que $100 \%$ após 12 meses quando comparado ao valor inicial de linfócitos totais. Os valores médios apresentaram um aumento de $7,45 \%$ após seis meses de imunoterapia e de 22,25\% após 12 meses. A análise estatística desta amostra se mostrou muito significativa com o teste ANOVA one way e com o teste " $t$ " de Student, especialmente após 12 meses. A Tabela 3 mostra as variações e análises estatísticas dos valores de linfócitos totais nos grupos analisados.

A avaliação dos valores basais da relação entre as subpopulações de linfócitos CD4 e CD8 não apresentou diferença significativa na comparação das amostras com e sem QT/RT ( $>>0,05)$ na análise pelo teste “ $t$ ” de Student, como nas anteriores.

Analisando os resultados dos pacientes submetidos à QT/RT, após seis meses de uso do extrato de timo, 20 pacientes $(66,66 \%)$ tiveram um aumento do valor da relação de linfócitos CD4 e CD8, enquanto que após 12 meses isto aconteceu em 27 casos (90\%). Comparando com o valor médio basal, estes dados representam uma elevação de 4,81\% após seis meses e de $31,02 \%$ após 12 meses. A análise estatística pelo teste ANOVA one way foi extremamente significativa, e o teste " $t$ " de Student mostrou relevância após 12 meses e entre

Tabela 2 - Variação dos leucócitos nos dois grupos (com e sem QT/RT).

\begin{tabular}{|c|c|c|c|}
\hline Com QT/RT & Valor & Sem QT/RT & Valor \\
\hline Média & & Média & \\
\hline basal & $5325,30 \quad( \pm 1259,78)$ & basal & $6043,50( \pm 1753,35)$ \\
\hline 6 meses & $5312,00 \quad( \pm 1463,65)$ & 6 meses & $6599,50( \pm 1908,80)$ \\
\hline 12 meses & $6517,66 \quad( \pm 1806,86)$ & 12 meses & $7290,00 \quad( \pm 1553,90)$ \\
\hline Teste $\mathbf{t}$ & & Teste $\mathbf{t}$ & \\
\hline Basal x 6m & $\mathrm{p}=0,96(\mathrm{NS})$ & Basal x 6m & $\mathrm{p}=0,34(\mathrm{NS})$ \\
\hline Basal x 12 m & $\mathrm{p}=0,004$ & Basal x 12 m & $\mathrm{p}=0,02$ \\
\hline $6 \mathrm{~m} \times 12 \mathrm{~m}$ & $\mathrm{p}=0,006$ & $6 \mathrm{~m} \times 12 \mathrm{~m}$ & $\mathrm{p}=0,04$ \\
\hline TesteANOVA & $\mathrm{p}=0,003$ & TesteANOVA & $\mathrm{p}=0,08$ \\
\hline Basal x 6m & & Basal x 6m & \\
\hline Basal x $12 \mathrm{~m}$ & muito significativo & Basal x 12 m & não significativo \\
\hline $6 \mathrm{~m} \times 12 \mathrm{~m}$ & & $6 \mathrm{mx} 12 \mathrm{~m}$ & \\
\hline \multicolumn{4}{|c|}{ Pós- teste Tukey-KramerPós- teste Tukey-Kramer } \\
\hline Basal x 6m & $\mathrm{p}>0,05(\mathrm{NS})$ & Basal x 6m & Não realizado por \\
\hline Basal x $12 \mathrm{~m}$ & $\mathrm{p}<0,01$ & Basal x $12 \mathrm{~m}$ & não ser significativo \\
\hline $6 \mathrm{mx} 12 \mathrm{~m}$ & $\mathrm{p}<0,01$ & $6 \mathrm{~m} \times 12 \mathrm{~m}$ & pelo teste ANOVA \\
\hline
\end{tabular}

Valores expressos em média \pm desvio padrão, valor de p, (NS) não significativo. 
Tabela 3 - Variação dos linfócitos totais nos dois grupos (com e sem QT/RT).

\begin{tabular}{|c|c|c|c|}
\hline Com QT/RT & Valor & Sem QT/RT & Valor \\
\hline Média & & Média & \\
\hline basal & $1714,20 \quad( \pm 475,21)$ & basal & $1882,40 \quad( \pm 417,10)$ \\
\hline 6 meses & $1832,93 \quad( \pm 493,31)$ & 6 meses & $2022,70 \quad( \pm 389,55)$ \\
\hline 12 meses & $2269,80 \quad( \pm 723,70)$ & 12 meses & $2301,20 \quad( \pm 583,91)$ \\
\hline Teste $\mathbf{t}$ & & Teste $\mathrm{t}$ & \\
\hline Basal x 6m & $\mathrm{p}>0,05(\mathrm{NS})$ & Basal x 6m & $\mathrm{p}>0,05(\mathrm{NS})$ \\
\hline Basal x $12 \mathrm{~m}$ & $\mathrm{p}<0,01$ & Basal x $12 \mathrm{~m}$ & $\mathrm{p}=0,01$ \\
\hline $6 \mathrm{~m} \times 12 \mathrm{~m}$ & $\mathrm{p}<0,05$ & $6 \mathrm{~m} \times 12 \mathrm{~m}$ & $\mathrm{p}=0,05$ \\
\hline TesteANOVA & $\mathrm{p}=0,008$ & TesteANOVA & $\mathrm{p}=0,02$ \\
\hline Basal x 6m & & Basal x $6 \mathrm{~m}$ & \\
\hline Basal x $12 \mathrm{~m}$ & muito significativo & Basal x $12 \mathrm{~m}$ & muito significativo \\
\hline $6 \mathrm{~m} \times 12 \mathrm{~m}$ & & $6 \mathrm{~m} \times 12 \mathrm{~m}$ & \\
\hline \multicolumn{4}{|c|}{ Pós- teste Tukey-KramerPós- teste Tukey-Kramer } \\
\hline Basal x 6m & $\mathrm{p}>0,05(\mathrm{NS})$ & Basal x $6 \mathrm{~m}$ & $\mathrm{p}>0,05(\mathrm{NS})$ \\
\hline Basal x $12 \mathrm{~m}$ & $\mathrm{p}<0,01$ & Basal x $12 \mathrm{~m}$ & $\mathrm{p}>0,05$ \\
\hline $6 \mathrm{~m} \times 12 \mathrm{~m}$ & $\mathrm{p}<0,05$ & $6 \mathrm{~m} \times 12 \mathrm{~m}$ & $\mathrm{p}>0,05(\mathrm{NS})$ \\
\hline
\end{tabular}

Valores expressos em média \pm desvio padrão, valor de p, (NS) não significativo.

6 e 12 meses. O pós-teste de comparações múltiplas de TukeyKramer confirmou estes resultados.

Com relação aos resultados do grupo não submetido à QT/RT, tanto aos seis como aos 12 meses, 13 pacientes (65\%) obtiveram um aumento do valor da relação CD4/CD8 em comparação com o inicial. Aos seis meses os valores médios da relação de linfócitos CD4 e CD8 apresentaram um aumento de $11,76 \%$ e de $28,82 \%$ aos 12 meses. Mesmo tendo ocorrido estes aumentos a análise estatística não se mostrou significativa $(\mathrm{p}>0,05)$ neste grupo com o teste " $t$ " $d e$ Student e pelo método ANOVA one way. Na Tabela 4 estão relacionados os resultados obtidos na avaliação da relação entre linfócitos CD4 e CD8 nos dois grupos e a análise estatística.

Tabela 4 - Resultados da relação de linfócitos CD4 e CD8 nos dois grupos (com e sem QT/RT).

\begin{tabular}{|c|c|c|c|}
\hline Com QT/RT & Valor & Sem QT/RT & Valor \\
\hline Média & & Média & \\
\hline basal & $(+0,85)$ & basal & $(+0,79)$ \\
\hline 6 meses & $( \pm 0,63)$ & 6 meses & $( \pm 1,08)$ \\
\hline 12 meses & $2,45 \quad( \pm 0,64)$ & 12 meses & $( \pm 1,08)$ \\
\hline Teste $\mathbf{t}$ & & Teste $t$ & \\
\hline Basal x 6m & $\mathrm{p}=0,05(\mathrm{NS})$ & Basal x 6m & $\mathrm{p}>0,05(\mathrm{NS})$ \\
\hline Basal x $12 \mathrm{~m}$ & $\mathrm{p}=0,004$ & Basal x $12 \mathrm{~m}$ & $\mathrm{p}>0,05(\mathrm{NS})$ \\
\hline $6 \mathrm{mx} 12 \mathrm{~m}$ & $\mathrm{p}=0,004$ & $6 \mathrm{~m} \times 12 \mathrm{~m}$ & $\mathrm{p}>0,05(\mathrm{NS})$ \\
\hline Teste ANOVA & $\mathrm{p}=0,0001$ & TesteANOVA & não significativo \\
\hline Basal x 6m & & Basal x 6m & \\
\hline Basal x $12 \mathrm{~m}$ & muito significativo & Basal x $12 \mathrm{~m}$ & \\
\hline $6 \mathrm{~m} \times 12 \mathrm{~m}$ & & $6 \mathrm{mx} 12 \mathrm{~m}$ & \\
\hline \multicolumn{4}{|c|}{ Pós- teste Tukey-KramerPós- teste Tukey-Kramer } \\
\hline Basal x 6m & $\mathrm{p}<0,001$ & Basal x $6 \mathrm{~m}$ & Não realizado por \\
\hline Basal x $12 \mathrm{~m}$ & $\mathrm{p}<0,001$ & Basal x $12 \mathrm{~m}$ & não ser significativo \\
\hline $6 \mathrm{~m} \times 12 \mathrm{~m}$ & $\mathrm{p}<0,01$ & $6 \mathrm{~m} \times 12 \mathrm{~m}$ & pelo teste ANOVA \\
\hline
\end{tabular}

Valores expressos em média \pm desvio padrão, valor de p, (NS) não significativo. 
Vale ressaltar que nenhum paciente referiu efeitos colaterais com o uso do extrato de timo, assim como também não houve relato da necessidade de interromper os ciclos de quimioterapia ou radioterapia pelo número baixo de leucócitos, linfócitos totais ou da relação entre linfócitos CD4 e CD8. Os efeitos imunossupressores da terapia antineoplásica foram minimizados com o uso da timulina.

\section{DISCUSSÃO}

A busca de melhores resultados em relação à taxa de sobrevida e qualidade de vida dos pacientes portadores de neoplasias malignas tem dado origem a vários trabalhos científicos. Inúmeras publicações têm destacado o papel da imunoterapia como mais uma opção terapêutica que visa a obtenção destes resultados ${ }^{11-16,32-35}$.

A imunossupressão é uma condição comumente encontrada em indivíduos com câncer, que acontece não só pela própria doença como também pelo comprometimento do estado nutricional e psicológico dos pacientes. Aliado a isto, o tratamento das neoplasias malignas que consiste basicamente na ressecção do tumor, quimioterapia e radioterapia, também interfere significativamente no funcionamento do sistema imune, que é essencial para a recuperação do paciente. Seria desta forma um somatório de condições que afetam negativamente a resposta imune com chances de comprometer $\mathrm{o}$ estado clínico do paciente. A manutenção da competência imunológica é fundamental, especialmente, para que a quimioterapia e a radioterapia não sofram interrupção em seus esquemas de aplicação.

A imunodeficiência é normalmente expressa por leucopenia, linfopenia, redução no número de células T e das subpopulações CD4 e CD8, além da inversão na relação entre linfócitos T helper e supressor. É também descrito que o acometimento da imunidade celular, em particular, está relacionado não apenas ao crescimento, mas também à disseminação da neoplasia ${ }^{32}$. Todas estas alterações comprometem o funcionamento do sistema imunológico levando a um estado de imunossupressão que favorece ao aparecimento de doenças oportunistas e infecciosas causando impacto na resposta clínica do paciente.

A associação da imunoterapia, como adjuvante, visa à restauração do sistema imune e à prevenção das complicações decorrentes da imunossupressão. Entre as diversas drogas imunomoduladores conhecidas, a timulina é uma que se destaca, havendo várias referências da sua aplicação no tratamento de doenças não neoplásicas ${ }^{7,11,21}$, neoplásicas ${ }^{16,20,25}$, com relatos maior tempo de sobrevida dos pacientes ${ }^{33}$ e remissão tumoral $^{36}$.

A timulina possui efeitos imunomoduladores quantitativos e qualitativos sobre os linfócitos T, caracterizando a sua ação sobre a imunidade celular. Esta linhagem de células é a mais afetada nas doenças malignas, sendo agravada com o uso da quimioterapia. Como a timosina é capaz de induzir a maturação e diferenciação dos linfócitos, ocorre uma melhora significativa em número e função destas células no sangue periférico. Este estímulo promove um efeito em cascata sobre as funções das células B, macrófagos, aumento da produção de Interleucina-2 e do número de seus receptores de alta afinidade, aumento da atividade das células natural killers (NK), da produção de interferon-gama, um efeito restaurador do índice de fagocitose, e aumento da capacidade lítica das células efetoras ${ }^{1,2,4,6,16,22,23}$. Todos estes dados caracterizam a função reguladora e moduladora da resposta imune produzida pela timulina.

Neste trabalho observamos um aumento no número de linfócitos em $90 \%$ dos pacientes submetidos à QT/RT e em $85 \%$ do grupo que não foi submetido à QT/RT. A normalização da relação entre os linfócitos CD4/CD8 foi conseguida em $90 \%$ dos pacientes submetidos à QT/RT e em $65 \%$ entre os que não fizeram QT/RT. Estes resultados caracterizam uma restauração da resposta imunológica, e estão de acordo com os estudos obtidos que referem menor redução na relação de linfócitos $\mathrm{CD} 4 / \mathrm{CD} 8$, menor neutropenia, aumento significativamente maior no número de células T, recuperação dos níveis normais de células $\mathrm{T}$, além de explorarem os possíveis efeitos protetores do extrato de timo sobre a linfopenia induzida pela quimioterapia e radioterapia.

Assim como é referido em vários trabalhos ${ }^{33,35,36}$, a administração de timulina minimizou os efeitos colaterais normalmente relacionados à quimioterapia e radioterapia, não havendo necessidade de interrupção destes tratamentos além dos pacientes referirem maior tolerância ao uso de doses mais elevadas das drogas antineoplásicas.

A timulina foi bem tolerada pelos pacientes não exibindo nenhum efeito colateral, corroborando os dados referidos na literatura ${ }^{16,20,33,35}$.

A análise destes resultados nos permite concluir que o uso da timulina foi capaz de restaurar os níveis das células T, equilibrar a relação entre as subpopulações dos linfócitos CD4 e CD8, promovendo desta forma a recuperação da competência imunológica. Houve uma grande diminuição na incidência de mielotoxicidade e os danos imunossupressores e colaterais induzidos pela terapia antineoplásica foram reduzidos, não havendo necessidade de interromper os ciclos destes tratamentos.

A timulina não exibiu nenhum efeito mutagênico e não mostrou nenhuma toxicidade mesmo quando usada em altas concentrações.

\section{AGRADECIMENTOS}

A autora agradece ao Laboratório Extratos Alergênicos Ltda pelo fornecimento da timulina, aos professores doutores Ricardo Marques Dias e Maria Lucia Pires pela ajuda com a análise estatística, a João Vargens e Helena Brascher pela realização dos exames complementares, a Luiz Carlos de Castro Pinto Garritano e Paloma Garrido pela ajuda na coleta e formatação dos dados, a Regina Chaloub e Eduardo Friedman pela revisão do texto em português e inglês, respectivamente. 


\begin{abstract}
Background: To evaluate the action of thymus extract (Thymulin) in patients diagnosed with neoplastic disease submitted to surgery treatment, with and without adjuvant chemotherapy and radiotherapy, comparing the results obtained in both groups after 6 and 12 months of treatment. Method: Retrospective study on 50 patients, with analyses on the variations of the leukocyte count, total lymphocytes and CD4/CD8 lymphocytes after 6 and 12 months after immunostimulation with Thymulin by subcutaneous via. Results: In the group submitted to QT/RT, there was an increase in the number of total leukocytes in 13 cases (43.33\%) after 6 months and in 25 cases (83.33\%) after 12 months. Regarding the number of lymphocytes, after 6 months of thymus extract use, 19 patients (63.33\%) presented higher levels of lymphocytes, and after 12 months, this occurred in 27 cases (90\%). The CD4/CD8 lymphocytes ratio demonstrated an increase after 6 months, in 20 patients (66.66\%) and, after 12 months in 27 cases (90\%). All these data presented statistical significance with the ANOVA test, being $p=0,003$ for the number of leukocytes, $p=0.0008$ for the number of total lymphocytes and $p<0.0001$ for the CD4/CD8 lymphocytes ratio. Regarding the group that was not submitted to QT/RT, the increase in leukocyte levels occurred after 6 months in 17 patients (85\%), and after 12 months in 18(90\%). In 2 cases (10\%) the leukocyte levels increased over $100 \%$ after 12 months. The number of lymphocytes increased in 12 patients (60\%) after 6 months, and in 17 (85\%) after 12 months. The CD4/CD8 lymphocytes ratio grew both after 6 and after 12 months in 13 patients (65\%). The statistical analysis of this sample proved to be important in the evaluation of the leukocytes and total lymphocytes with Student's t-test after 12 months, and it was very significant $(p=0.02)$ concerning the total lymphocytes count made with the ANOVA test. The analysis of the variations in the CD4/CD8 lymphocytes ratio levels was not significant, even though, there was an increase on those values. It was not necessary to interrupt de QT/RT cycles and no patient complained about intolerance in using Thymulin. Conclusion: The use of Thymulin associated with QT/RT was capable of restoring and keeping the immune response in control and reducing the immunosupressor and collateral damages induced by antineoplastic therapy without any side effects.
\end{abstract}

Key words: Thymus extracts; Neoplasms; Antineoplastic agents.

\section{REFERÊNCIAS}

1. Benedikt H. The use of thymus supplementation: a novel approach to immune regulation. The Original Internist. 2000; 7(1):29-32.

2. Mazzoli AB. Factores hormonales timicos. In: Actualizaciones en imunologia basica y aplicada. 1974:179-81.

3. Lengyel G, Feher J. Thymostimulin in clinical practice. Orv Hetil. 1994; 135:(52):2871-5.

4. Lin CY, Low TL. A comparative study on the immunological effect of bovine and porcine thymic extracts: induction of lymphoproliferative response and enhacement of interleukin-2, gamma-interferon and tumor necrotic factor production in vitro on cord blood lymphocytes. Immunopharmacology. 1989; 18:110.

5. Santarelli L, Di Lorenzo L, Valentino M, Bracci M, Rapisarda V, Cassano F, Elia G, Martino MG, Neri G, Apostoli P, Soleo L. Ridotta produzione di timulina in corso di esposizione professionale a piombo. G Ital Med Lav Erg. 2005; 27(Suppl 1):68-72.

6. Tzehoval E, SZtein MB, Goldstein AL. Thymosins alpha 1 and beta 4 potentiate the antigen-presenting capacity of macrophages. Immunopharmacology. 1989; 18(2):107-13.

7. Balikin VF, Uchaikin VF, Konev VA, Iusuf-Zade AA. Clinicopathogenetic role of hormones of the pituitary-adrenal system and somatotropin in the development of immunosuppression in chronic hepatitis B and delta infection in children and the approach to its correction. Pediatriia. 1991; (10):39-44.

8. Dworniak D, Tchorzewski H, Pokoca L, Tkacz B, Drobnik S, Baj Z, Luciak M. Treatment with thymic extract TFX for chronic active hepatitis B. Arch Immunol Ther Exp (Warsz). 1991; 39(56):537-47.

9. Garcia-Contreras F, Nevárez-Sida A, Constantino-Casas P, AbudBastida F, Garduño-Espinosa J. Cost-effectiveness of chronic hepatitis $\mathrm{C}$ treatment with thymosin alpha-1. Arch Med Res. 2006; 37(5):663-73.

10. Braga PC, Dal Sasso M, Maci S, Piatti G, Palmieri R, Bruno L, Albanese C. Restoration of polymorphonuclear leukocyte function in elderly subjects by thymomodulin. J Chemother. 1994; 6(5):354-9.

11. Eckert K, Schmitt M, Garbin F, Wahn U, Maurer HR. Thymosin alpha 1 effects, in vitro, on lymphokine-activated killer cells from patients with primary immunodeficiencies: preliminary results. Int J Immunopharmacol. 1994; 16(12):1019-25.

12. Blanca Maria MM, Pérez Gerardo L. Uma antigua aliada: la timomodulina. Alergia Imunologia Pediatr. 2000;9(2):65-8.

13. Iino S, Toyota J, Kumada H, Kiyosawa K, Kakumu S, Sata M, Suzuki H, Martins EB. The efficacy and safety of thymosin alpha-1 in Japanese patients with chronic hepatitis B: results from a randomized clinical trial. J Viral Hepat. 2005; 12(3):300-6.

14. Martin P, Friedman LS. Therapies for hepatitis B virus: current status and future possibilities. Adv Exp Med Biol. 1992; 312:111-20.

15. Rustgi VK. Thymalfasin for the treatment of chronic hepatitis $C$ infection. Expert Rev Anti Infec Ther. 2005; 3(6):885-92.

16. Alba E, Visentin L, Farina C, Wierdis T. Prevention of infection and improvement of cenesthesia with thymostimulin during chemotherapy following mastectomy. Minerva Ginecol. 1991; 43(12):585-7.

17. Denaro A, Stivala F, Mazzarino MC. Immunologic study on patients with head and neck cancer treated with thymopentin associated with surgery, chemotherapy and radiotherapy. Acta Otorhinolaryngol Ital. 1994; 14(6):611-25.

18. Luraschi A, Saglietti G, Fedeli P, Gioria A, Borgotti P. Partial remission of non-Hodgkin's lymphoma (stage IV-B) following immunostimulating therapy with thymopentin. Minerva Med. 1992; 83(6):377-80.

19. Munno I, Marinaro M, Gesario A, Cannuscio B, Michel Y, Paulling E. Immunomodulatory effects of alpha interferon and thymostimulin in patients with neoplasias. Clin Diagn Lab Immunol. 1995; 2(4):503-5.

20. Negri L, Calabrese F, Correggia F, Miozzo S, Giacomasso S. Chemotherapy and thymostimulin in the treatment of advancedstage breast neoplasms. Minerva Med. 1992; 83(5):283-8.

21. Ricci E, Giaccone M, Pecchio F, Lale-Murix E, De Simone M, Nano M. Immunological variations after thymic stimulation with special reference to Il2-rs in patients operated for neoplasms. Minerva Chir. 1991; 46(10):507-10. 
22. Vuckovic-Dekic Lj, Susnjar S, Stanojevic-Bakic N, Rajner L. Immunomodulating effect of thymus protein fraction on disorders of general immunity in patients with lung carcinoma. Glas Srp Akad Nauka [Med]. 1994; (44):63-70.

23. Balleari E, Timitilli S, Musselli C, Ghio R. In vivo hemopoietic activity of thymic extract "Thymustimulin" in aged healthy humans. Thymus. 1992;19(1):59-63.

24. Batian AN, Abramovskaia AK, Batian ON. Prevention of side effects of specific chemotherapy in patients with pulmonary tuberculosis. Probl Tuberk. 1991; (7):60-2.

25. Braga M, Di Francesco A, Gianotti L, Baccari P, Di Carlo V. Perioperative administration of thymopentin for the prevention of infections in oncologic surgery. Minerva Chir. 1992; 47(34):125-30.

26. Lai N, Lavosi V, Pinna S, Sallis G, Colombo E, Vargiu P. Postoperative infections: the use of thymostimulin (TP1) in patients at risk. G Chir. 1992; 13:(6-7):377-8.

27. Manfè AZ, Grigoletto R, de Silvestro G, Bernante, Faccioli AM. Efficacy of immunomodulating treatment in patients with different degrees of immunosuppression. Minerva Chir. 1991; 46(10):5115.

28. Serra GE, Gustavino C, Serra Cervetti GG. Proposal for the treatment of cervix dysplasia with immunomodulators. Minerva Ginecol. 1992; 44(1-2):15-8.

29. Wiley DJ, Douglas J, Beutner K, Cox T, Fife K, Moscicki AB, Fukumoto L. External genital warts: diagnosis, treatment and prevention. Clin Infect Dis. 2002; 35(suppl 2):S210-24.

30. Goldstein G. Isolation of bovine thymin: a polypeptide hormone of the thymus. Nature. 1974; 247(5435):11-4.

31. Goldstein AL, Guha A, Zatz MM, Hardy MA, White A. Purification and biological activity of thymosin, a hormone of the thymus gland. Proc Natl Acad Sci USA. 1972; 69(7):1800-3.
32. Berkow R, Beers MH, Fletcher AJ, editors. The merck manual of medical information: home edition. Whitehouse Station, N.J: Merck Research Labs; 1997.

33. Macchiarini P, Danesi R, Del Tacca M, Angeletti CA. Effects of thymostimulin on Chemotherapy-induced toxicity and long-term survival in small cell lung cancer patients. Anticancer Res. 1989; 9(1):193-6.

34. Iaffaioli RV, Frasci G, Tortora G, Ciardiello F, Nuzzo F, Scala S, Pacelli R, Bianco AR. Effect thymic extract thymostimulin on the incidence of infections and mielotoxicity during adjuvant chemotherapy for breast cancer. Thymus. 1988;12(2):69-75.

35. Mustacchi, G. High-dose Folinic acid (FA) and Fluorouracil (FU) or minus Thymostimulin (TS) for treatment of metastatic colorectal cancer: Results of a randomized multicentric clinical trial. Anticancer Res. 1994; 4(2):149-55.

36. McCormack MP, Rabbitts TH. Activation of the T-cell oncogene LMO2 after gene therapy for X-linked severe combined immunodeficiency. N Engl J Med. 2004; 350(9):913-22.

Como citar este artigo:

Garritano CR. Avaliação do uso de extrato de timo (timulina) em pacientes com neoplasia maligna submetidos ao tratamento cirúrgico. Rev Col Bras Cir. 2007; 34(4). Disponível em URL: www.scielo.br/rcbc

Endereço para correspondência:

Célia Regina de Oliveira Garritano

Praça Saens Pena, 45/1410. Tijuca.

20520-090 - Rio de Janeiro - RJ.

Telefone: xx (21) 2264-5552.

e-mail: cgarritano@oi.com.br 